OFFICIAL JOURNAL OF THE JAPANESE CIRCULATION SOCIETY

In 2011 (January to December), the average time for primary review for all original manuscripts submitted

to Circulation Journal was $\mathbf{1 2 . 0}$ days.

\title{
Message From the Editor-in-Chief
}

Editorial Statistics and Best Reviewers Award for 2011

Hiroaki Shimokawa

\section{Cardiology Societies in the Asian/Pacific Region}

The Chinese Society of Cardiology

Dayi Hu

\section{Reviews}

Contemporary Medical Management of Systolic Heart Failure Ajith P. Nair, Tendoh Timoh, Valentin Fuster

Hemodynamic Classifications of Acute Heart Failure and Their Clinical Application - An Update Sunu S. Thomas, Anju Nohria

Options to Overcome Clopidogrel Response Variability Kyung Woo Park, Hyo-Soo Kim

\section{Editorials}

Region- and Condition-Dependence of the Membrane and $\mathrm{Ca}^{2+}$ Clocks in the Sinus Node Junko Kurokawa, Tetsushi Furukawa

Cardiac Sympathetic Activity and Left Ventricular Dyssynchrony - An Old and New Issue Shinichi Fujimoto

Progress in the Functional Assessment of Human Coronary Collateral Circulation Masatoshi Fujita, Shoichi Miyamoto

The Less Embraces the Greater in Detecting Multiple Coronary Artery Disease Shoji Sanada, Masafumi Kitakaze, Issei Komuro

Effect and Safety of Landiolol in Patients With Acute Myocardial Infarction Undergoing Primary Percutaneous Coronary Intervention

Minoru Yoshiyama

Multicenter Study of the Prevalence and Distribution of Sarcometric Gene Mutations in Familial Hypertrophic Cardiomyopathy - A Milestone for Genetic Diagnosis in the Japanese Population Shinichi Niwano

Can Acute Myocardial Infarction Sneak Out From Takotsubo? Makoto Hirai

Percutaneous Transluminal Pulmonary Angioplasty for Distal-Type Chronic Thromboembolic Pulmonary Hypertension 


\section{Arrhythmia/Electrophysiology}

Selective Sinoatrial Node Optical Mapping and the Mechanism of Sinus Rate Acceleration Tetsuji Shinohara, Hyung-Wook Park, Boyoung Joung, Mitsunori Maruyama, Su-Kiat Chua, Seongwook Han, Mark J. Shen, Peng-Sheng Chen, Shien-Fong Lin

Anticoagulation Control Quality Affects the D-Dimer Levels of Atrial Fibrillation Patients Yosuke Nakatani, Koichi Mizumaki, Kunihiro Nishida, Tadakazu Hirai, Masao Sakabe, Yoshitaka Oda, Shuji Joho, Akira Fujiki, Takashi Nozawa, Hiroshi Inoue

Epicardial Ablation With Irrigated Electrodes - Effect of Bipolar vs. Unipolar Ablation on Lesion Formation Koichi Nagashima, Ichiro Watanabe, Yasuo Okumura, Kazumasa Sonoda, Masayoshi Kofune, Hiroaki Mano, Kimie Ohkubo, Toshiko Nakai, Satoshi Kunimoto, Yuji Kasamaki, Atsushi Hirayama

\section{Cardiovascular Intervention}

Predicting Long-Term Mortality After First Coronary Revascularization - The Kyoto Model Shiro Tanaka, Ryuzo Sakata, Akira Marui, Yutaka Furukawa, Toru Kita, Takeshi Kimura on behalf of the CREDO-Kyoto Investigators

Comparison of Clinical Outcome of Infrapopliteal Angioplasty Between Korean Diabetic and Non-Diabetic

Patients With Critical Limb Ischemia Hyeon Min Ryu, Jung-Sun Kim, Young Guk Ko, Myeong-Ki Hong, Yangsoo Jang, Dong-Hoon Choi

Spatial Distribution and Temporal Evolution of Scattering Centers by Optical Coherence Tomography in the Poly(L-Lactide) Backbone of a Bioresorbable Vascular Scaffold Juan Luis Gutiérrez-Chico, Maria D. Radu, Roberto Diletti, Alexander Sheehy, Mary Beth Kossuth, James P. Oberhauser, Thierry Glauser, Joel Harrington, Richard J. Rapoza, Yoshinobu Onuma, Patrick W. Serruys

Sirolimus-Eluting Stent vs. Everolimus-Eluting Stent for Coronary Intervention in Patients on Chronic Hemodialysis

Takashi Sakakibara, Hideki Ishii, Takanobu Toriyama, Toru Aoyama, Hiroshi Takahashi, Daisuke Kamoi, Yoshihiro Kawamura, Kazuhiro Kawashima, Kohei Yoneda, Tetsuya Amano, Miho Tanaka, Daiji Yoshikawa, Mutsuharu Hayashi, Tatsuaki Matsubara, Toyoaki Murohara

\section{Cardiovascular Surgery}

Procedure- and Age-Specific Risk Stratification of Single Aortic Valve Replacement in Elderly Patients Based on Japan Adult Cardiovascular Surgery Database Nobuhiro Handa, Hiroaki Miyata, Noboru Motomura, Takeshi Nishina, Shinichi Takamoto, The Japan Adult Cardiovascular Database Organization

Mid-Term Outcomes After Aortic Valve Replacement With the 17-mm St. Jude Medical Regent Valve Homare Okamura, Atsushi Yamaguchi, Hiroshi Nagano, Satoshi Itoh, Hideki Morita, Kazuhiro Naito, Koichi Yuri, Hideo Adachi

Initial Experience of Conversion of Toyobo Paracorporeal Left Ventricular Assist Device to DuraHeart

Left Ventricular Assist Device Daisuke Yoshioka, Taichi Sakaguchi, Shunsuke Saito, Shigeru Miyagawa, Hiroyuki Nishi, Yasushi Yoshikawa, Satsuki Fukushima, Takayoshi Ueno, Toru Kuratani, Yoshiki Sawa

Surgical Treatment for Ellis Type 3 Coronary Perforation During Percutaneous Catheter Intervention Toshinori Totsugawa, Masahiko Kuinose, Hidenori Yoshitaka, Keijiro Katayama, Yoshimasa Tsushima, Atsuhisa Ishida, Genta Chikazawa, Arudo Hiraoka

\section{Heart Failure}

Effect of Left Ventricular Dyssynchrony on Cardiac Sympathetic Activity in Heart Failure Patients With Wide QRS Duration Hidekazu Tanaka, Kazuhiro Tatsumi, Sei Fujiwara, Takayuki Tsuji, Akihiro Kaneko, Keiko Ryo, Yuko Fukuda, Kensuke Matsumoto, Mayumi Shigeru, Akihiro Yoshida, Hiroya Kawai, Ken-ichi Hirata 


\section{Imaging}

In Vivo Optical Coherence Tomography of Very Late Drug-Eluting Stent Thrombosis Compared With Late In-Stent Restenosis

Shinichiro Miyazaki, Yoshikazu Hiasa, Takefumi Takahashi, Yudai Yano, Tomoko Minami, Naotsugu Murakami, Michiko Mizobe, Yohei Tobetto, Takafumi Nakagawa, Po-Min Chen, Riyo Ogura, Hitoshi Miyajima, Kenichiro Yuba, Shinobu Hosokawa, Koichi Kishi, Ryuji Ohtani

Cardiac lodine-123 Metaiodobenzylguanidine (123|-MIBG) Scintigraphy Parameter Predicts Cardiac and

Cerebrovascular Events in Type 2 Diabetic Patients Without Structural Heart Disease Kunio Yufu, Naohiko Takahashi, Norihiro Okada, Tetsuji Shinohara, Mikiko Nakagawa, Masahide Hara, Hironobu Yoshimatsu, Tetsunori Saikawa

\section{Ischemic Heart Disease}

Efficacy of Clotinab in Acute Myocardial Infarction Trial-ST Elevation Myocardial Infarction (ECLAT-STEMI) Jung-Sun Kim, Sang-Min Park, Byeong-Keuk Kim, Young-Guk Ko, Donghoon Choi, Myeong-Ki Hong, In Whan Seong, Byung Ok Kim, Hyeon-Cheol Gwon, Bum Kee Hong, Seung-Jae Tahk, Seong-Wook Park, Chong Jin Kim, Myung-Ho Jeong, Junghan Yoon, Yangsoo Jang for the ECLAT-STEMI Trial investigators

Novel Acute Collateral Flow Index in Patients With Total Coronary Artery Occlusion During ST-Elevation Myocardial Infarction

Simcha R. Meisel, Michael Shochat, Aaron Frimerman, Aya Asif, David S. Blondheim, Jacob Shani, Yoseph Rozenman, Avraham Shotan

Ratio of Serum n-3 to n-6 Polyunsaturated Fatty Acids and the Incidence of Major Adverse Cardiac

Events in Patients Undergoing Percutaneous Coronary Intervention

Takenori Domei, Hiroyoshi Yokoi, Shoichi Kuramitsu, Yoshimitsu Soga, Takeshi Arita, Kenji Ando, Shinichi Shirai, Katsuhiro Kondo, Koyu Sakai, Masahiko Goya, Masashi Iwabuchi, Masayuki Ueeda, Masakiyo Nobuyoshi

Postischemic Myocardial Stunning Is Superior to Transient Ischemic Dilation for Detecting Multivessel Coronary Artery Disease

Satoshi Hida, Taishiro Chikamori, Hirokazu Tanaka, Yuko Igarashi, Chie Shiba, Tsuguhisa Hatano, Yasuhiro Usui, Akira Yamashina

Randomized Study on the Efficacy and Safety of Landiolol, an Ultra-Short-Acting $\beta 1$-Adrenergic Blocker,

in Patients With Acute Myocardial Infarction Undergoing Primary Percutaneous Coronary Intervention Kenji Hanada, Takumi Higuma, Fumie Nishizaki, Takanori Sukekawa, Takashi Yokota, Masahiro Yamada, Shin Saito, Motoi Kushibiki, Koichi Oikawa, Naoki Abe, Hirofumi Tomita, Tomohiro Osanai, Ken Okumura

\section{Molecular Cardiology}

Additive Effect of ANRIL and BRAP Polymorphisms on Ankle-Brachial Index in a Taiwanese Population Pei-Chien Tsai, Yi-Chu Liao, Tsung-Hsien Lin, Edward Hsi, Yi-Hsin Yang, Suh-Hang Hank Juo

\section{Myocardial Disease}

Prevalence and Distribution of Sarcomeric Gene Mutations in Japanese Patients With Familial Hypertrophic Cardiomyopathy

Haruna Otsuka, Takuro Arimura, Tadaaki Abe, Hiroya Kawai, Yoshiyasu Aizawa, Toru Kubo, Hiroaki Kitaoka, Hiroshi Nakamura, Kazufumi Nakamura, Hiroshi Okamoto, Fukiko Ichida, Mamoru Ayusawa, Shinichi Nunoda, Mitsuaki Isobe, Masunori Matsuzaki, Yoshinori L. Doi, Keiichi Fukuda, Taishi Sasaoka, Toru Izumi, Naoto Ashizawa, Akinori Kimura

Differences in Negative T Waves Between Takotsubo Cardiomyopathy and Reperfused Anterior Acute Myocardial Infarction

Masami Kosuge, Toshiaki Ebina, Kiyoshi Hibi, Noriaki Iwahashi, Kengo Tsukahara, Mitsuaki Endo, Nobuhiko Maejima, Zenko Nagashima, Hiroyuki Suzuki, Satoshi Morita, Satoshi Umemura, Kazuo Kimura

\section{Pediatric Cardiology and Adult Congenital Heart Disease}

Evaluation of Transplacental Treatment for Fetal Congenital Bradyarrhythmia - Nationwide Survey in Japan -

Takekazu Miyoshi, Yasuki Maeno, Haruhiko Sago, Noboru Inamura, Satoshi Yasukohchi, Motoyoshi Kawataki, Hitoshi Horigome, Hitoshi Yoda, Mio Taketazu, Makio Shozu, Motoki Nii, Hitoshi Kato, Satoshi Hayashi, Asako Hagiwara, Akiko Omoto, Wataru Shimizu, Isao Shiraishi, Heima Sakaguchi, Kunihiro Nishimura, Keiko Ueda, Shinji Katsuragi, Tomoaki Ikeda 


\section{Vascular Medicine}

VE-Cadherin low $a$-Smooth Muscle Actin+ Component of Vascular Progenitor Cells Correlates With the Coronary Artery Gensini Score

Chao-Hung Wang, I-Chang Hsieh, Shih-Jen Chen, Jong-Shyan Wang, Wen-Jin Cherng, Chun-Chi Chen, lii-Jan Lam, Shing-Jong Lin

\section{Rapid Communication}

Percutaneous Transluminal Pulmonary Angioplasty Markedly Improves Pulmonary Hemodynamics and

Long-Term Prognosis in Patients With Chronic Thromboembolic Pulmonary Hypertension

Koichiro Sugimura, Yoshihiro Fukumoto, Kimio Satoh, Kotaro Nochioka, Yutaka Miura, Tatsuo Aoki, Shunsuke

Tatebe, Saori Miyamichi-Yamamoto, Hiroaki Shimokawa

\section{JCS Guidelines}

Guidelines for Risks and Prevention of Sudden Cardiac Death (JCS 2010) - Digest Version JCS Joint Working Group

Guidelines for the Clinical Use of 24 Hour Ambulatory Blood Pressure Monitoring (ABPM) (JCS 2010)

- Digest Version -

JCS Joint Working Group

\section{Letters to the Editor}

Some Caution When Conducting Long-Term Follow-up Study of Cardiovascular Mortality

(Letter) Tomoyuki Kawada

(Reply) Johanna M. Geleijnse, Yuji Hirai, Daan Kromhout

\section{Corrigendum}

